\title{
Computer Simulation of Three Particles Sedimentation in a Narrow Channel
}

\author{
Rongqian Chen, Yi Liu, and Deming Nie \\ Institute of Fluid Mechanics, China Jiliang University, Hangzhou 310018, China \\ Correspondence should be addressed to Deming Nie; nieinhz@gmail.com
}

Received 3 December 2016; Revised 3 February 2017; Accepted 28 February 2017; Published 15 March 2017

Academic Editor: Claudia Adduce

Copyright (C) 2017 Rongqian Chen et al. This is an open access article distributed under the Creative Commons Attribution License, which permits unrestricted use, distribution, and reproduction in any medium, provided the original work is properly cited.

The settling of three particles in a narrow channel is simulated via the lattice Boltzmann direct-forcing/fictitious domain (LBDF/FD) method for the Reynolds number ranging from 5 to 200. The effects of the wall and the Reynolds number are studied. It is interesting to find that at certain Reynolds numbers the left (right) particle is settling at 0.175 (0.825) of the channel width irrespective of its initial position or the channel width. Moreover, numerical results have shown that the lateral particles lead at small Reynolds numbers, while the central particle leads at large Reynolds numbers due to the combined effects of particle-particle and particle-wall interactions. The central particle will leave the lateral ones behind when the Reynolds number is large enough. Finally the effect of the Reynolds number on the trajectory of the lateral particles is presented.

\section{Introduction}

Particle sedimentation is one of the most common phenomena in nature and industrial production, such as fluidized beds, gas filtration technology, and sand deposition, which always presents a classical problem in hydrodynamic fields. It is important to understand the particle-particle interactions as well as the particle-fluid interactions to provide an insight into the behavior of particles suspended in fluids. To study the mechanism of particle sedimentation, a direct numerical simulation (DNS) is usually adopted. In a DNS method, the fluid flow and particle motion are coupled to study the dynamics of individual particles suspended in fluids, which is the highestresolution numerical method without any empirical model.

It is very important to deal with the curve boundary of particles in the simulation via the DNS method. Roughly speaking, there are two kinds of schemes which are usually adopted to simulate the particulate flows: the immersed boundary method $[1,2]$ and the fictitious domain method $[3,4]$. The essence of these two schemes is similar. In both schemes, a Lagrangian mesh is used to deal with the solids while an Eulerian mesh is used to treat the fluids. The nonslip condition is enforced by applying a forcing term into the Navier-Stokes equations. In comparison with conventional numerical methods such as the finite volume method and finite-element method, the computational efficiency is improved significantly because there is no requirement of remeshing procedure for the above-mentioned methods. The lattice Boltzmann direct-forcing/fictitious domain (LBDF/FD) method, which has been proposed by Nie and Lin [5], has been successfully applied to simulate the motion of two-dimensional and three-dimensional particles [6-8].

So far there exists some research work which focused on the study of the settling particle. Feng et al. [9] used the DNS method to simulate the sedimentation of circular and elliptical particles in a Newtonian fluid. They revealed five different falling patterns depending on the Reynolds number: steady motion with and without overshoot and weak, strong, and irregular oscillations. Feng et al. [9] also reported the occurrence of Drafting-Kissing-Tumbling (DKT), which is an important phenomenon in the particle sedimentation. Xia et al. [10] adopted a lattice Boltzmann method (LBM) to simulate the sedimentation of an elliptical particle in a narrow channel. They found five distinct modes according to the blockage ratio: oscillating, tumbling along the wall, vertical sedimentation, horizontal sedimentation, and an inclined mode. The situation may become more complex when multiple particles are taken into account. Aidun and Ding [11] simulated the sedimentation of two circular particles in an infinite channel by using LBM. Their work showed 


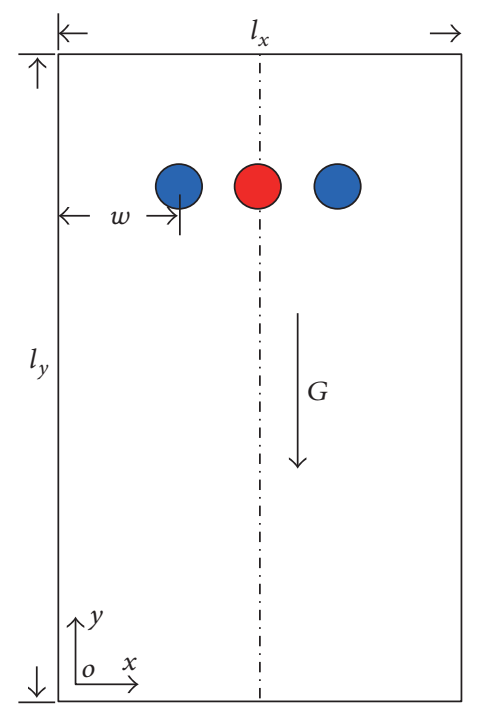

FIGURE 1: Schematic of the problem.

that the particles go through a complex transition to reach a low-dimensional chaotic state [11] due to the interaction between particles and the interaction between particles and walls. El Yacoubi et al. [12] simulated the sedimentation of multiple particles at an intermediate Reynolds number by using an immersed interface method. Some interesting falling patterns of particles were observed in their study. The middle particle is always leading in the case of odd-numbered particles, and the falling pattern is concave-up, whereas it is concave-down in the case of even-numbered particles [12]. Similarly, Nie et al. [13] studied the sedimentation of multiple particles via the LB-DF/FD method. They focused on the effect of the interaction between particles on the DKT process. Wang et al. [14] used the LBM to simulate the DKT phenomenon of two nonidentical particles. They [14] demonstrated that the effect of the diameter difference on the DKT process was significant. The two particles may not undergo the DKT process if the smaller particle is initially located above the larger one [14]. Recently, Nie et al. [15] reported the grouping behaviors of multiple particles settling along their line-of-centers in a narrow channel. They showed that the settling particles separated into several groups resulting from the particle-particle interaction, with each group settling at the same velocity. Furthermore, their work demonstrated that this type of grouping behavior strongly depended on the number of particles and the Reynolds number [15]. Verjus et al. [16] studied the sedimentation of two circular particles in a narrow channel and revealed some new features of the settling behavior of particles. They established the link between the terminal Reynolds number and the nondimensional driving force which displays various behaviors. More recently, Amiri Delouei et al. [17] developed a direct-forcing immersed boundary non-Newtonian lattice Boltzmann method to simulate the motion of particles in shear-thinning and shear-thickening fluids. They studied the DKT motion in non-Newtonian fluids for the first time, which shows that increasing the shear-thickening behavior of fluid leads to a significant increase in the kissing time.
However, the attention paid to the effect of particle-wall interaction on the motion of particles is very limited. It is unclear how the particles interact with each other when the hydrodynamic interaction between particles as well as particlewall interaction is taken into account. This motivates the present work, because it is expected that the effect of walls on the falling pattern of particles is significant. Furthermore, this problem could be more complicated and unexpected if the effect of inertia is taken into account. We may wish to inquire whether the middle particle is always leading in the case of odd-numbered particles. We may also wish to know under what circumstances the particles are falling together in the channel. Therefore, the purpose of this work is to simulate the sedimentation of three circular particles in a vertical channel by using the LB-DF/FD method. We focus on the effects of the Reynolds number as well as the walls on the falling pattern of particles and intend to provide a better understanding of the combined effects of particle-particle and particle-wall interactions on the stable or unstable motion of particles by considering the effect of inertia. We also aim to state that the particle sedimentation system is simple but rich in dynamics and worthy of extensive examination.

\section{Numerical Model}

The details of the LB-DF/FD method are presented elsewhere [5]. The physical model is shown in Figure 1, where the computational region is $l_{x} \times l_{y}$, and the density and diameter of the particles are $\rho_{p}$ and $d$, respectively. The distance between the lateral particles (the left particle and the right one) and the side wall is $w$. The three particles in a horizontal arrangement with zero initial velocity are released in a vertical channel. The velocity scale in the simulations is expressed as follows:

$$
U=\sqrt{\frac{\pi d\left(\rho_{r}-1\right) g}{2}},
$$

where $g$ is gravity and $\rho_{r}$ is the density ratio of solid particles and fluids, which is $\rho_{r}=\rho_{p} / \rho_{f}$. Therefore, the time scale 


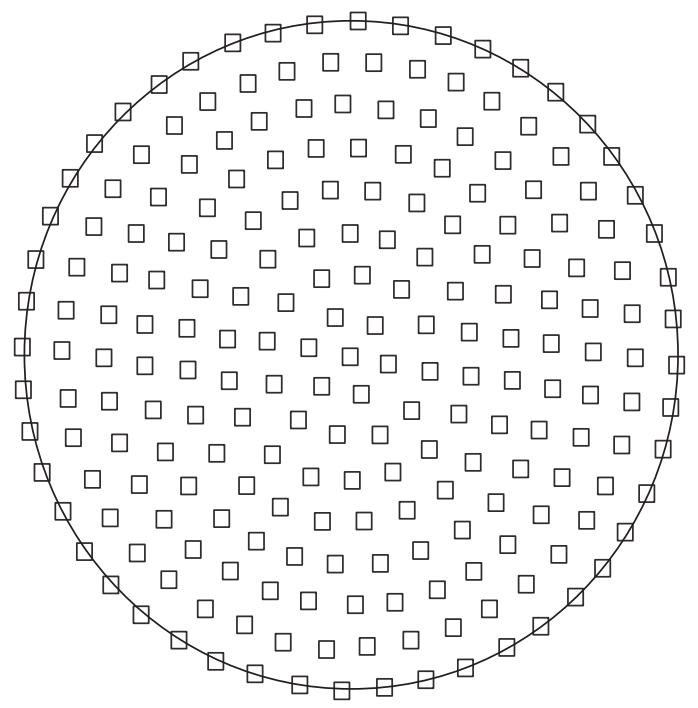

FigURE 2: Arrangements of Lagrangian points for circular particle.

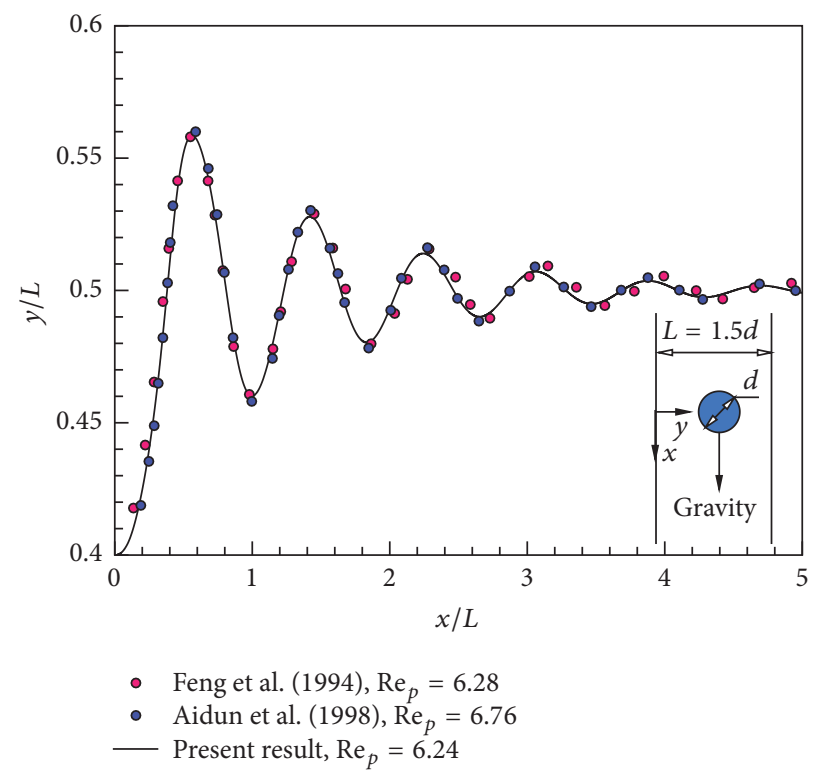

FIGURE 3: The trajectory of a circular particle settling in a vertical channel.

and Reynolds number are expressed as $T=d / U$ and $\operatorname{Re}=$ $U d / v$, respectively, where $v$ is the kinematic viscosity of the fluid. For convenience of description, some nondimensional parameters are introduced: $L_{x}=l_{x} / d, L_{y}=l_{y} / d$, and $W=$ $w / l_{x}$. The horizontal and vertical coordinates of particles are expressed as follows: $X=x / l_{x}$ and $Y=y / l_{y}$. The parameters are chosen as follows: $d=32, \rho_{p}=1.5$, and $\rho_{f}=1$ (in lattice unit). The height of the channel is fixed at $L_{y}=60$. The simulation starts at $5 d$ away from the top wall and ends when the distance between the center particle and the bottom wall is $5 d$. For convenience no-slip boundary conditions are set on all four fixed walls of the domain. This avoids specifying the far-field boundary conditions on a finite computational domain. The arrangement of Lagrangian points inside the circular particle is shown in Figure 2, with one point at the particle's center and $6 i$ points on the $i$ th ring.

\section{Validation}

The sedimentation of a circular particle in a narrow channel is adopted to validate the present computation method. The conditions used here are the same as those used by Feng et al. [9]. A circular particle with diameter $d=120$ and density $\rho_{p}=1.3$ is initially located at the centerline of the channel and then settled under gravity, as shown in Figure 3. The channel width is $L=1.5 d$. The particle trajectory is illustrated in Figure 3 along with other published results. The Reynolds number based on the particle diameter and terminal velocity is determined as 6.24. As can be seen, the present result agrees with the finite-element result [9] and LBM result [18]. It can be inferred from the figure that the particle moves away from the wall and undergoes a damping oscillation about the centerline of the channel until it reaches a steady state. 

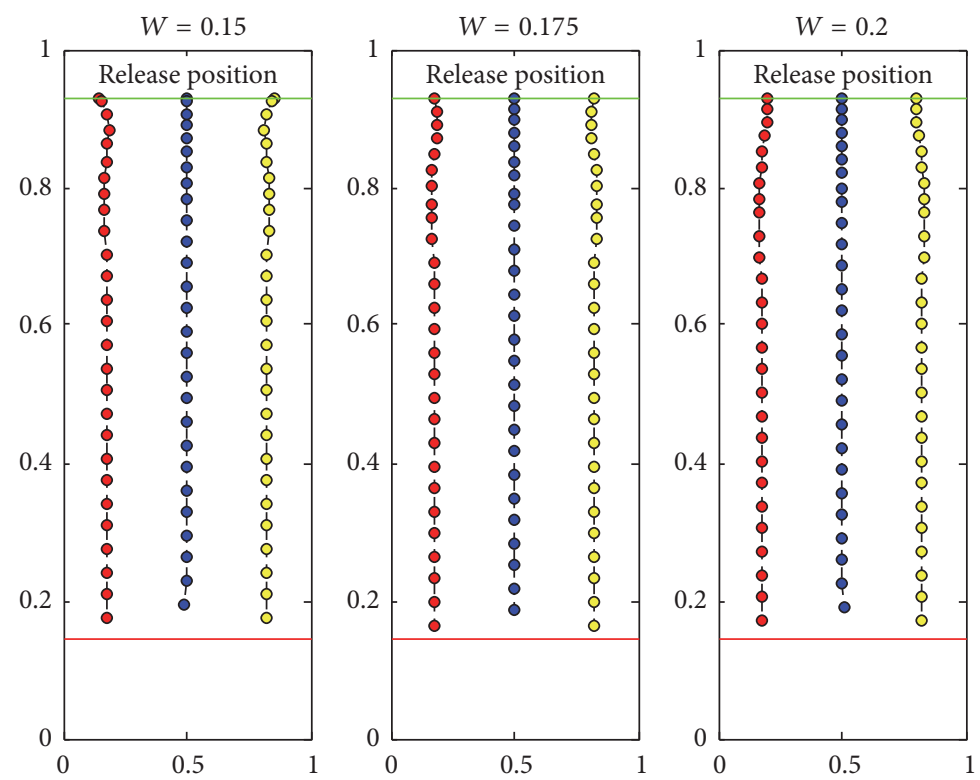

(a)

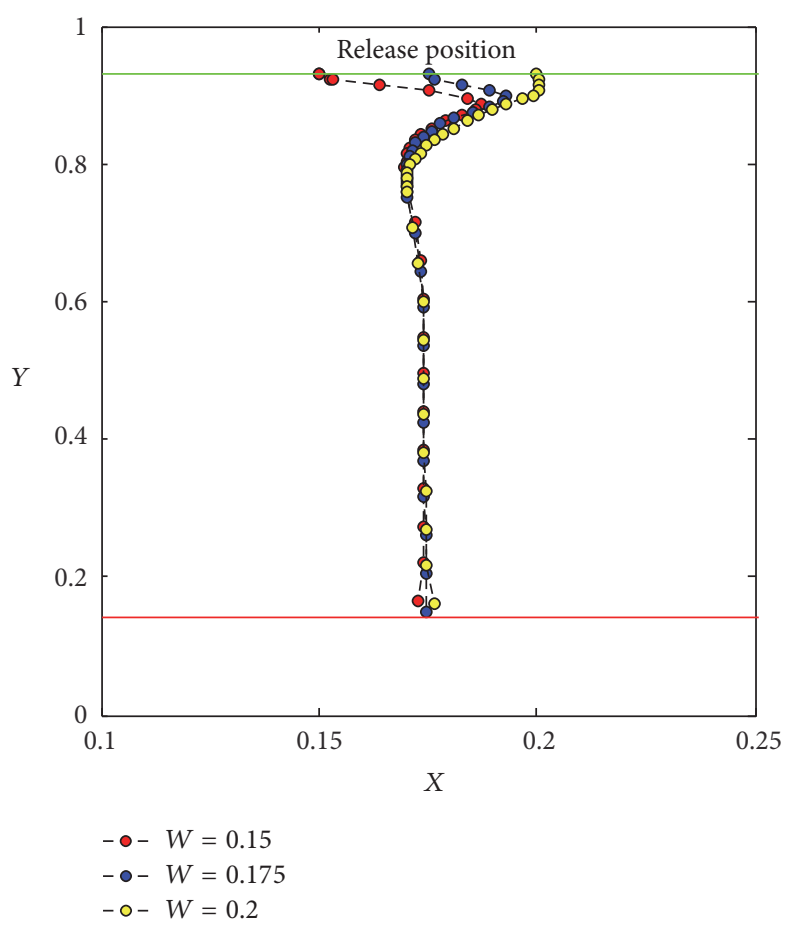

(b)

FIGURE 4: (a) The falling pattern for $L_{x}=8$ and $\mathrm{Re}=10$ and (b) the trajectory of the left particle for different initial positions at Re $=10$.

\section{Results and Discussion}

4.1. Effect of the Wall. First, we consider the effect of the wall as well as the value of $W$ on the falling pattern of the particles. For each case studied in this section, the width of the channel is fixed at $L_{x}=8$ unless specified otherwise. Three sets of the Reynolds number are taken into account: $\operatorname{Re}=10,80$, and 200. The effect of the wall is firstly examined by varying the value of $W$, which is the initial distance between the wall and the lateral particles.
Figure 4(a) shows the falling pattern for different value of $W$ at $\operatorname{Re}=10$. Generally speaking, the falling pattern is similar for all three $W$, as one can see in Figure 4(a). The flow is symmetrical with respect to the channel centerline. The concave-up of particle arrangement is observed, which suggests that the lateral particles lead while the central one trails when the Reynolds number is small. This is different from the observation made by El Yacoubi et al. [12]. In addition, numerical results also show that all the particles are settling at the same velocity finally, which is owing to the 

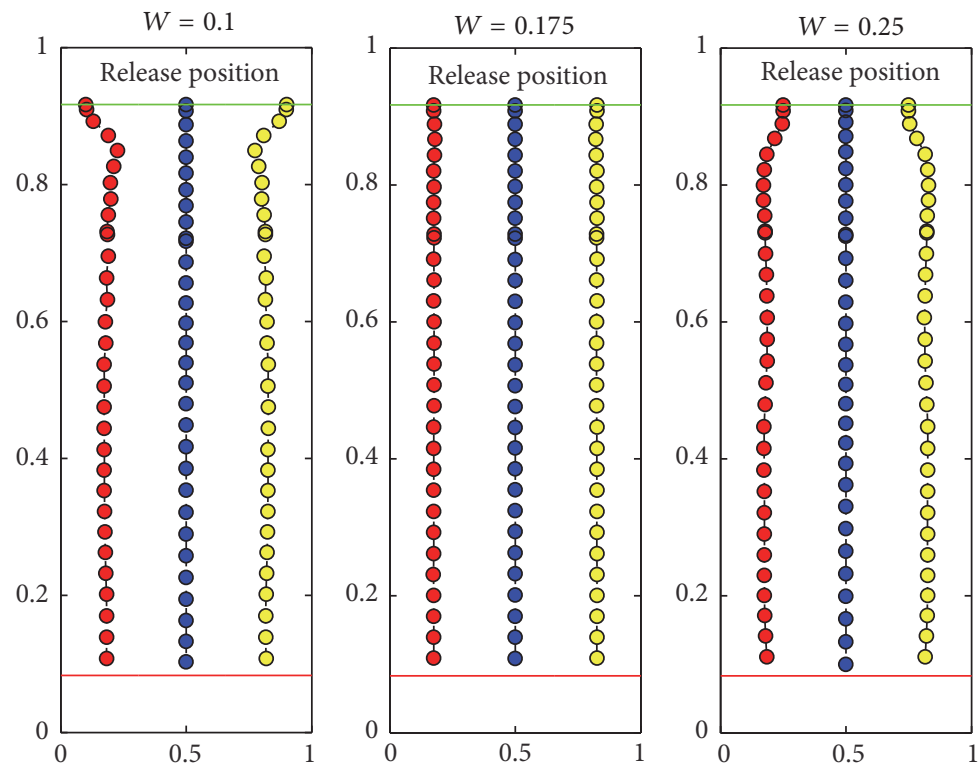

(a)

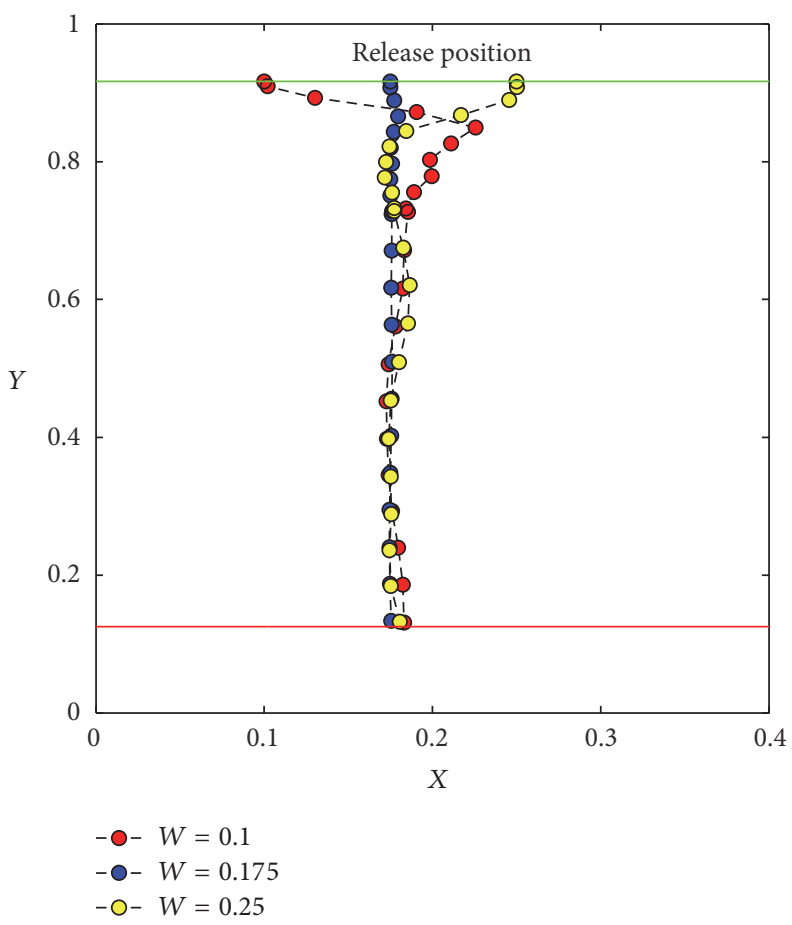

(b)

FIgURE 5: (a) The falling pattern for $L_{x}=8$ and $\mathrm{Re}=100$ and (b) the trajectory of the left particle for different initial positions at $\mathrm{Re}=100$.

effect of the walls. This will be further discussed in the next section. Figure 4(b) shows the effect of $W$ on the trajectory of the left particle, which indicates that the left particle is always settling at about 0.175 of the channel width irrespective of the value of $W$

Figure 5(a) shows the falling pattern for different value of $W$ at $\mathrm{Re}=100$. In comparison with the results of $\mathrm{Re}=$ 10 shown in Figure 4(a), one difference can be found in the falling pattern. The central particle is now leading while the lateral ones are trailing, as one can see in Figure 5(a). This becomes much more significant when the Reynolds number is larger, as shown in Figure 6(a), which presents the falling pattern at $\mathrm{Re}=200$. The central particle leaves the lateral ones behind. As a consequence of the influence of the wake, the lateral ones are moving towards the channel centerline, which is about 0.4 of the channel width eventually, much larger than that of $\operatorname{Re}=10$ or $\operatorname{Re}=100$. Furthermore, the particles are no longer moving together at $\operatorname{Re}=200$ because the central 

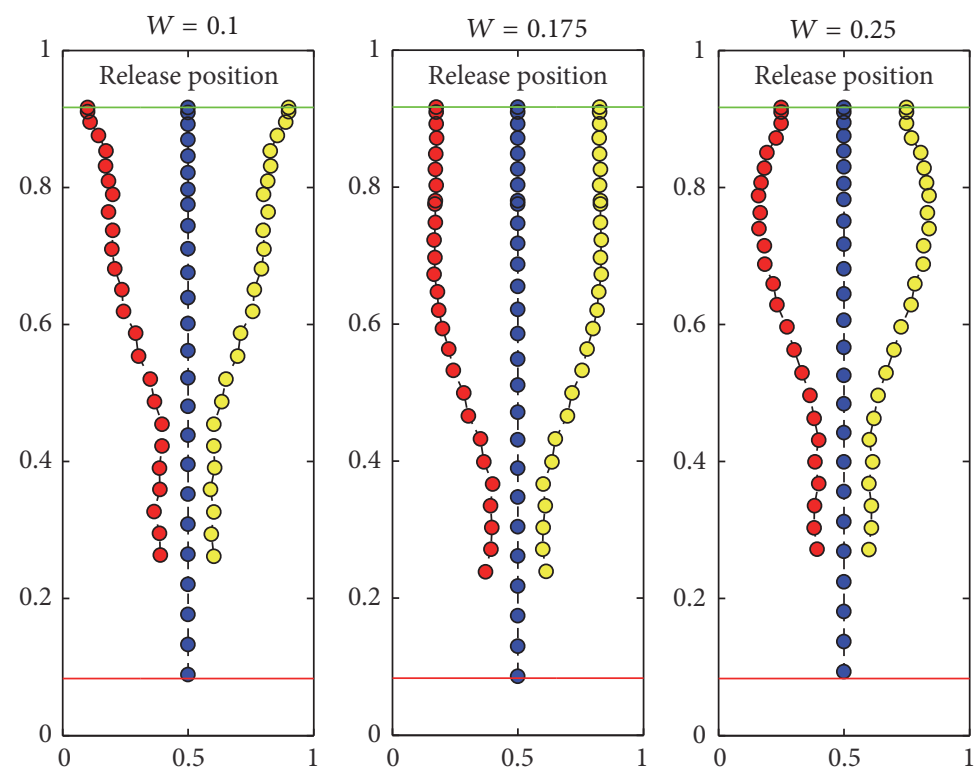

(a)

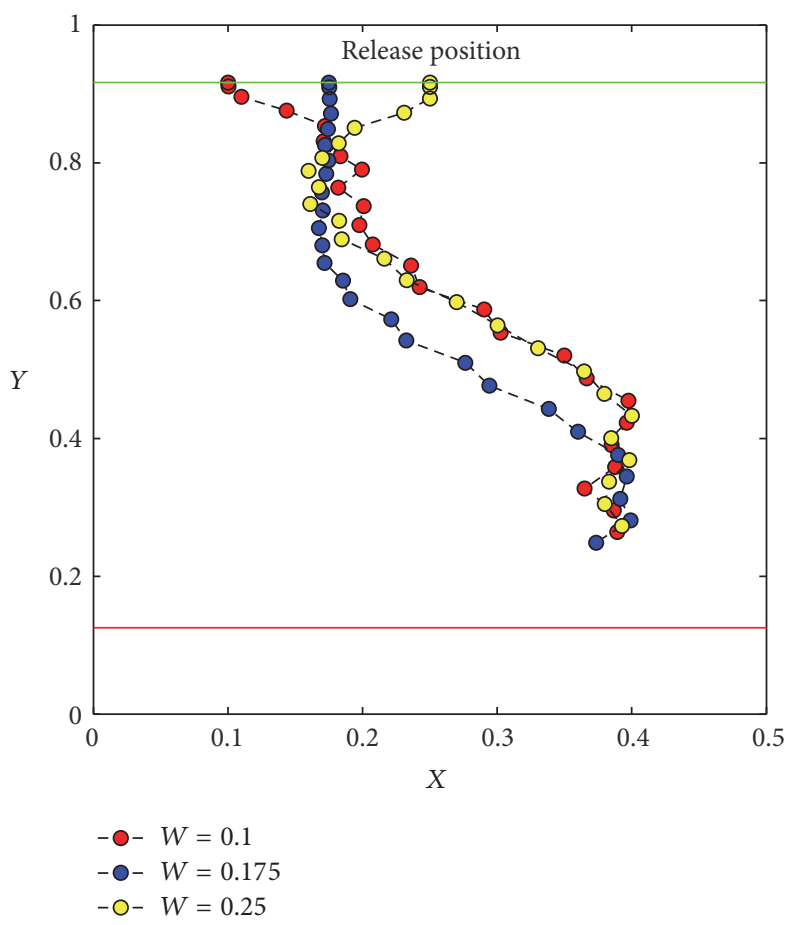

(b)

FIGURE 6: (a) The falling pattern for $L_{x}=8$ and $\mathrm{Re}=200$ and (b) the trajectory of the left particle for different initial positions at Re $=200$.

particle settles individually and has a larger velocity, which is different from the results of $\mathrm{Re}=10$ or $\mathrm{Re}=100$. This will be discussed in the next section. From Figures $4-6$, it can be inferred that the effect of the initial distance between the lateral particle and the wall on the falling pattern is negligible.

To examine the effect of the channel width, three kinds of channel are taken into account: $L_{x}=6,8$, and 10. Other parameters are fixed at $\operatorname{Re}=80$ and $W=0.175$. The results are shown in Figure 7. The falling pattern is similar for all results studied. It is interesting to find that the left particle always settles at about 0.175 of the channel width irrespective of the value of $L_{x}$, as shown in Figure 7(b). Figure 8 shows the instantaneous vorticity contour for different times at $\mathrm{Re}$ $=200$. It is observed that the vortex shedding takes place for the lateral particles when $t / T \geq 20$. As a result, the settling of the lateral particles is slowing down, leading to the fact that the central particle leaves them behind eventually. 

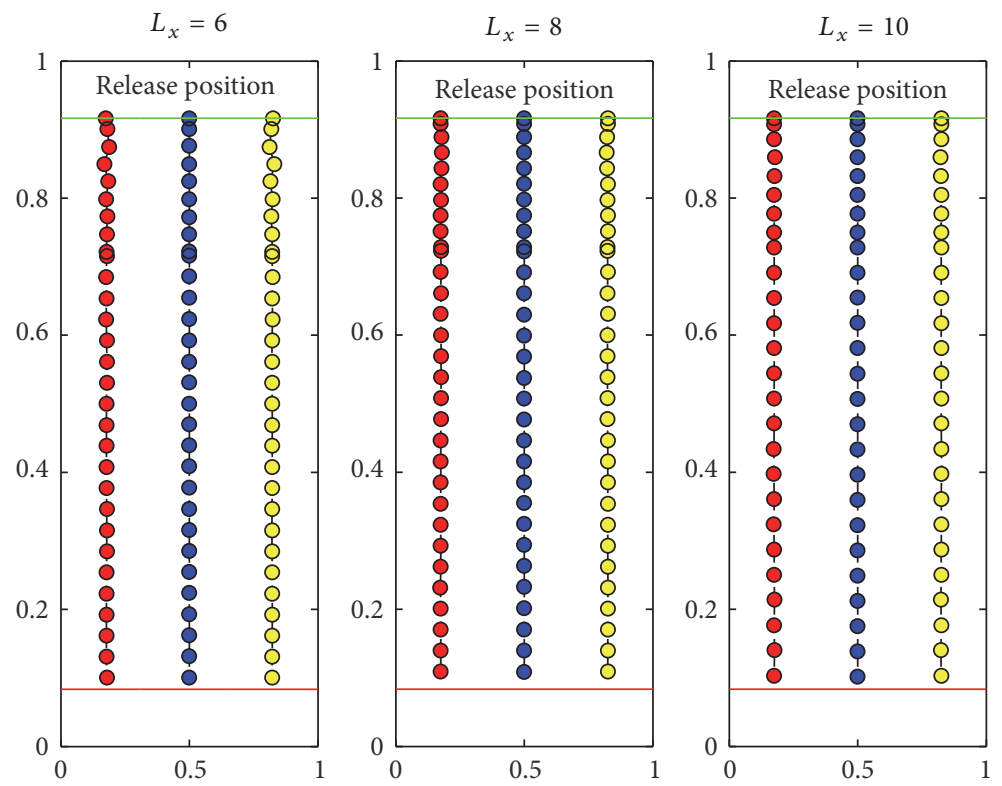

(a)

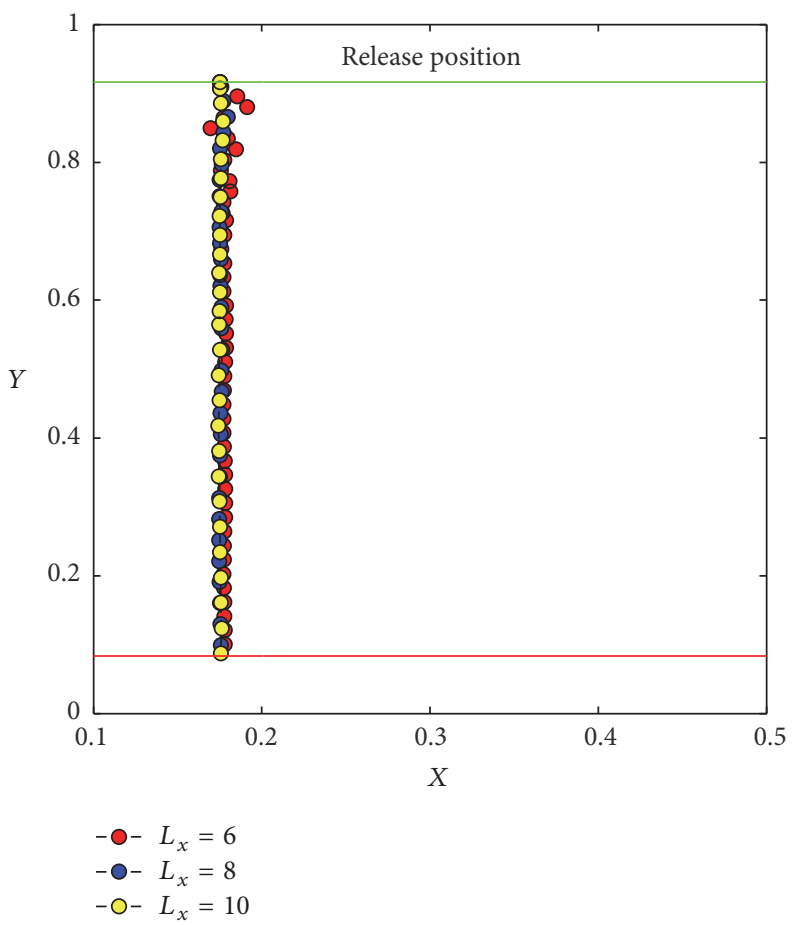

(b)

Figure 7: (a) The falling pattern for $W=0.175$ and $\mathrm{Re}=80$ and (b) the trajectory of the left particle for different channel width at Re $=80$.

4.2. Effect of the Reynolds Number. In this section, the effect of the Reynolds number on the settling of particles is examined. To quantitatively study the particle motion, the time evolution of the vertical position $(Y)$ and the settling velocity $\left(U_{y}\right)$ is shown in Figure 9 for $\operatorname{Re}=10,80,100$, and 200. The value of $\Delta Y=Y$ (lateral) $-Y$ (central) and $\Delta U_{y}=U_{y}$ (lateral) $-U_{y}$ (central) is also presented. As shown in Figures 9(a)-9(c), the settling velocity of the left particle is the same as that of the central one because $\Delta U_{y} \approx 0$ is seen for all three results $(\operatorname{Re}=10,80$, and 100$)$. In addition, it is obviously observed that $\Delta Y>0$ for $\operatorname{Re}=10$ and $\Delta Y<0$ for $\operatorname{Re}=100$, which represents the concave-down and concave-up pattern, respectively. In particular, all three particles are settling nearly in a horizontal line for $\mathrm{Re}=80$ because of $\Delta Y \approx 0$, as shown in Figure 9(b). On the other hand, the situation is different for $\mathrm{Re}=200$. The particles are settling at the same velocity during 


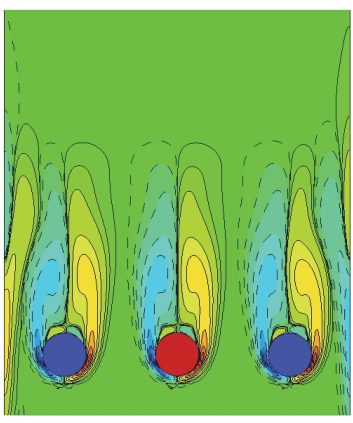

(a) $t / T=10$

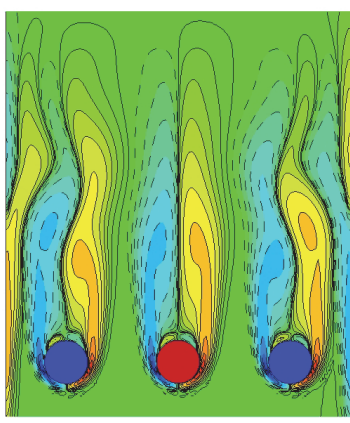

(b) $t / T=15$

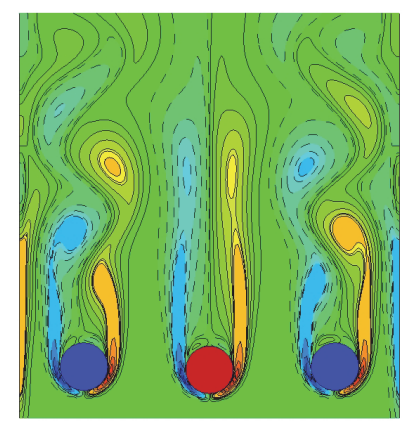

(c) $t / T=20$

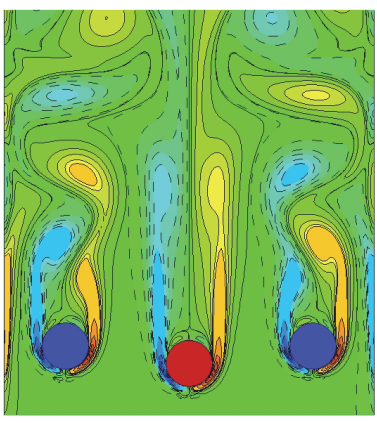

(d) $t / T=25$

FIgURE 8: Instantaneous vorticity contour for different times at $\mathrm{Re}=200$ and $W=0.175$.

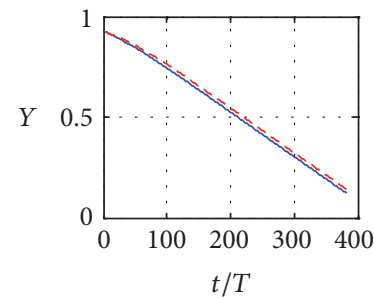

- Left particle

- - Central particle
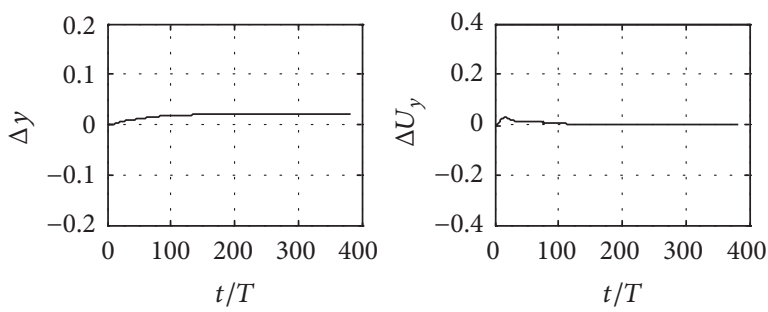

(a)

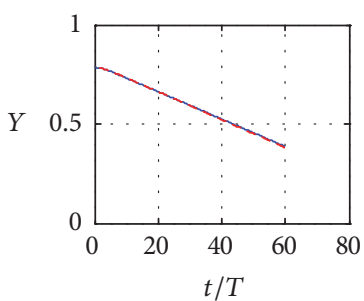

- Left particle

- - Central particle
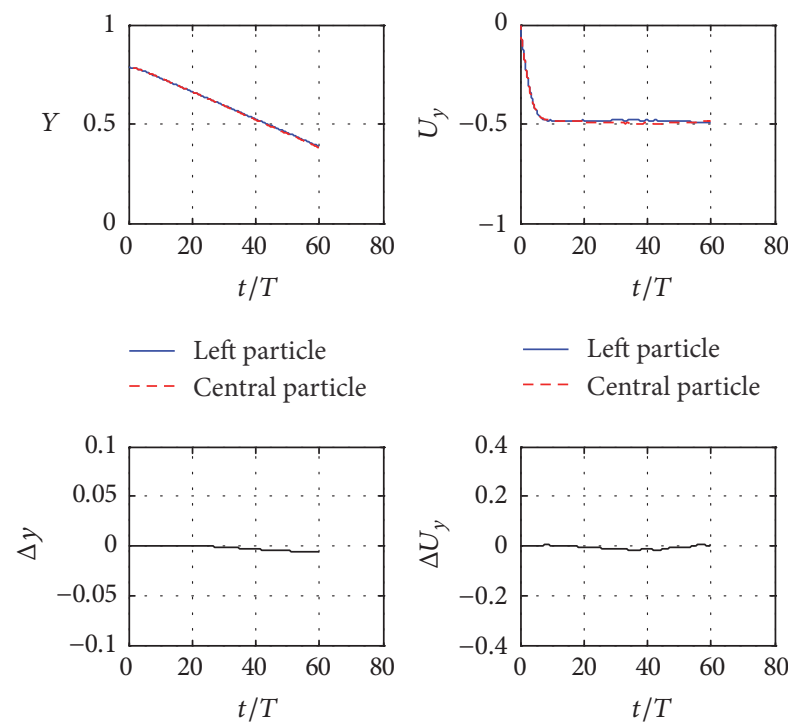

_ Left particle

- - Central particle

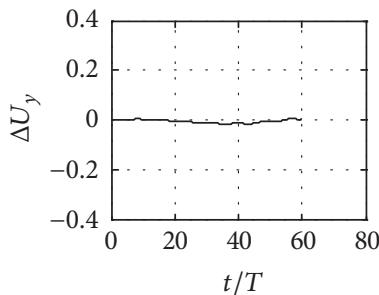

(c)
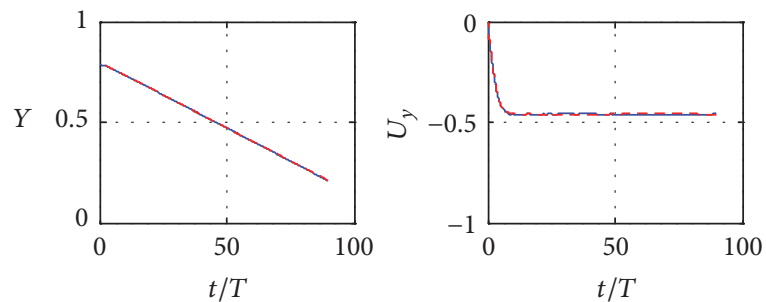

_ Left particle

- - Central particle

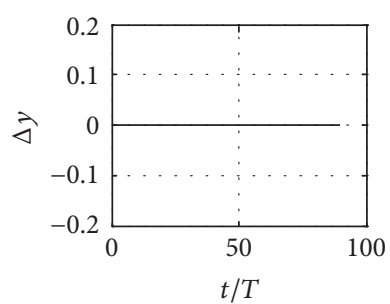

- - - Central particle

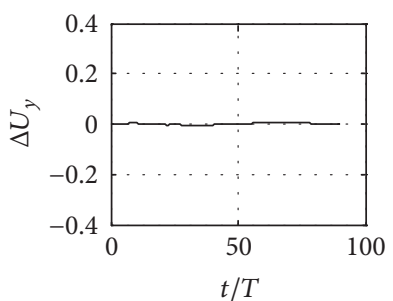

(b)
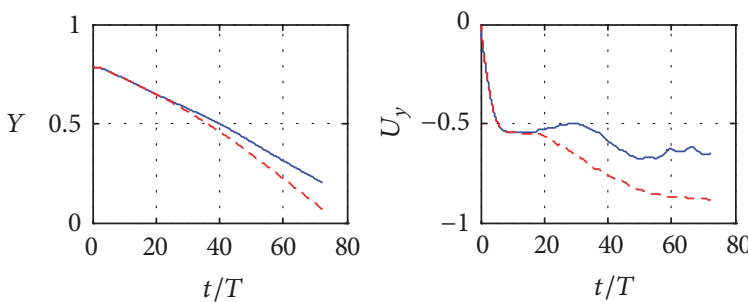

_ Left particle

- - Central particle

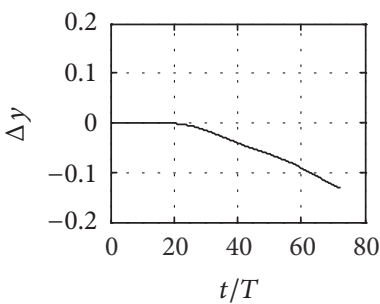

_ Left particle

- - - Central particle

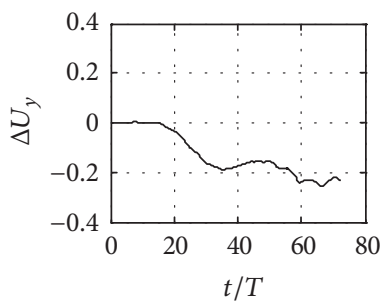

(d)

Figure 9: The motion of particles at different Reynolds numbers: (a) $\operatorname{Re}=10$, (b) $\operatorname{Re}=80$, (c) $\operatorname{Re}=100$, and (d) $\operatorname{Re}=200$. 


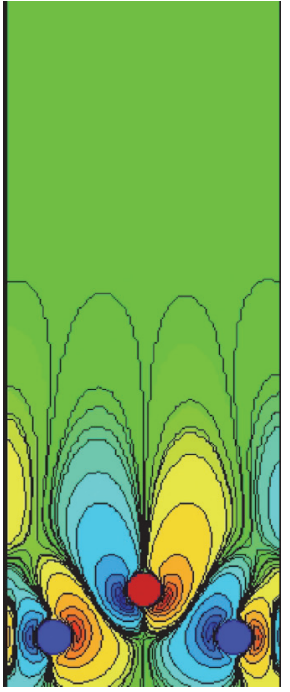

(a)

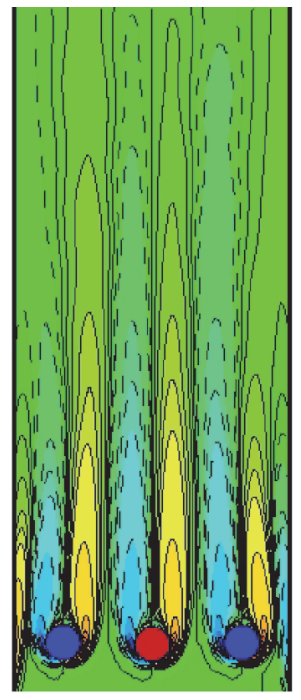

(b)

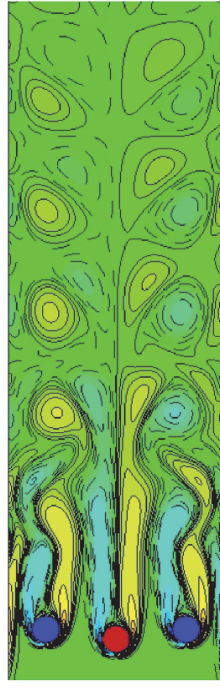

(c)

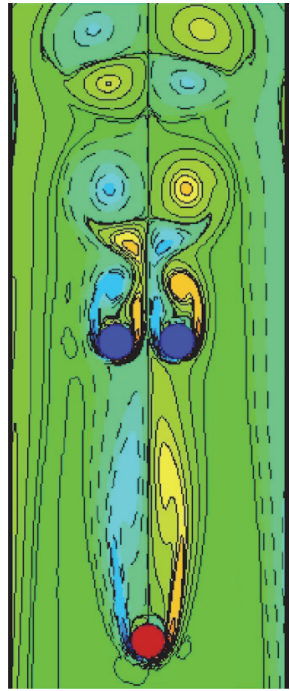

(d)

FIGURE 10: Instantaneous vorticity contour for different Reynolds numbers: (a) $\operatorname{Re}=10$, (b) $\operatorname{Re}=80$, (c) $\operatorname{Re}=100$, and (d) $\operatorname{Re}=200$.

the initial transients $(t / T<20)$. After that the central particle is settling at a faster velocity. As a result the distance between the central particle and the lateral ones $(\Delta Y)$ is increasing, which also demonstrates that the settling of particles is unsteady.

Figure 10 shows the instantaneous vorticity contour at different Reynolds numbers, displaying totally four falling patterns in the simulations. For $\mathrm{Re}=100$ shown in Figure 10(c), the vortex shedding is observed for all three particles, but the particles are settling together in the channel, which is different in the case of $\mathrm{Re}=200$.

In order to present more insight into the effect of the Reynolds number, numerous simulations have been carried out for the Reynolds number ranging from 5 to 200. Because of the symmetry of the flow, only the trajectory of the left particle is shown in Figure 11. The initial distance between the lateral particles and the walls is fixed at $W=0.175$.

The effect of the Reynolds number is significant, as can be seen in Figure 11. When the Reynolds number is small $(\mathrm{Re}<$ 10), the horizontal position of the left particle is $X_{0}<0.175$, which can be seen in Figure 11(a). Note that $X_{0}=0.175$ at $\operatorname{Re}=10$. Moreover, the left particle moves closer to the channel centerline as increasing Re. For instance, the value of $X_{0}$ is about 0.2 when $\mathrm{Re}=20$, as shown in Figure 11(b). This suggests that the wall repulsion is significant when Re is small. However, if Re is increasing further the left particle moves towards the left wall, instead. The reason behind this is that the mutual repulsion between particles becomes significant as increasing Re. For $40<\mathrm{Re} \leq 100$, it can be observed that the left particle is settling at about $X_{0}=0.175$ irrespective of the Reynolds number, as shown in Figure 11(d). When the Reynolds number is large enough $(\operatorname{Re} \geq 110)$, the left particle is unstable due to the vortex shedding, which moves closer to the channel centerline as Re is increasing.

\section{Conclusion}

In this paper, the LB-DF/FD method has been adopted to simulate the sedimentation of three particles in a narrow channel. The effects of the wall and the Reynolds number on particle trajectories have been studied. The present method is validated by simulating the sedimentation of a circular particle in a narrow channel.

Results have shown that at certain Reynolds numbers (Re $=10$ or $40<\operatorname{Re} \leq 100)$ the left particle always sediments at 0.175 of the channel width irrespective of its initial position or the channel width. In addition, results have also shown that the lateral particles lead at small Reynolds numbers, while the central particle leads at large Reynolds numbers. This indicates that the concave-down pattern as well as the concave-up pattern can be observed in the simulations. Results also show that the particles may settle in a horizontal line at intermediate Reynolds numbers, such as $\mathrm{Re}=80$. Furthermore, it has been found that when Re is small $(5<$ $\operatorname{Re} \leq 20)$ the lateral particles move closer to the channel centerline as Re increases. The opposite is true when Re is large $(20<\operatorname{Re} \leq 40)$. When $\operatorname{Re}$ is large enough $(\operatorname{Re} \geq 110)$, the lateral particles are oscillating due to the vortex shedding, which moves closer to the channel centerline as increasing Re.

\section{Conflicts of Interest}

The authors declare that they have no conflicts of interest. 


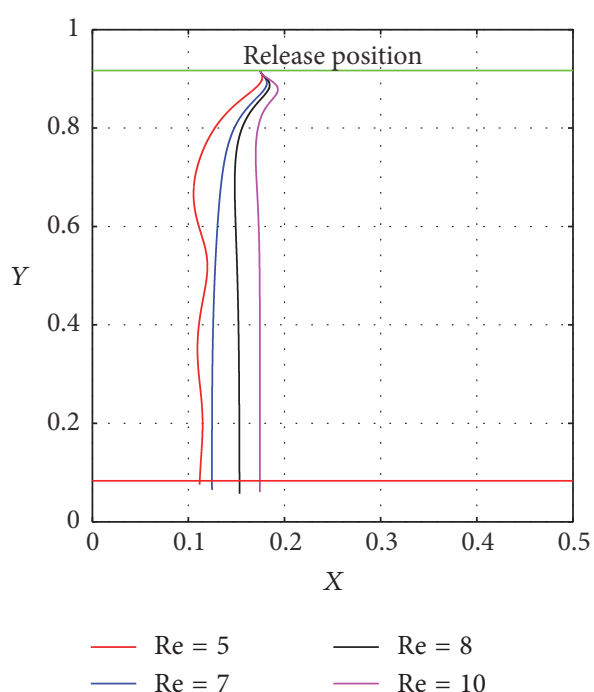

(a)

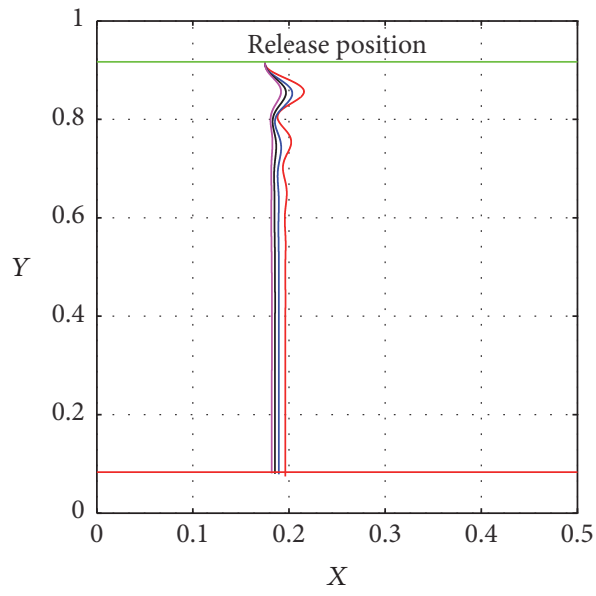

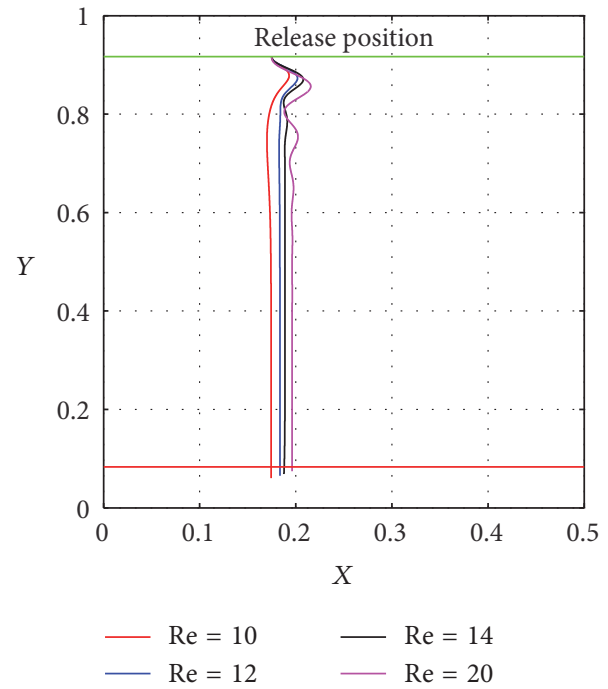

(b)
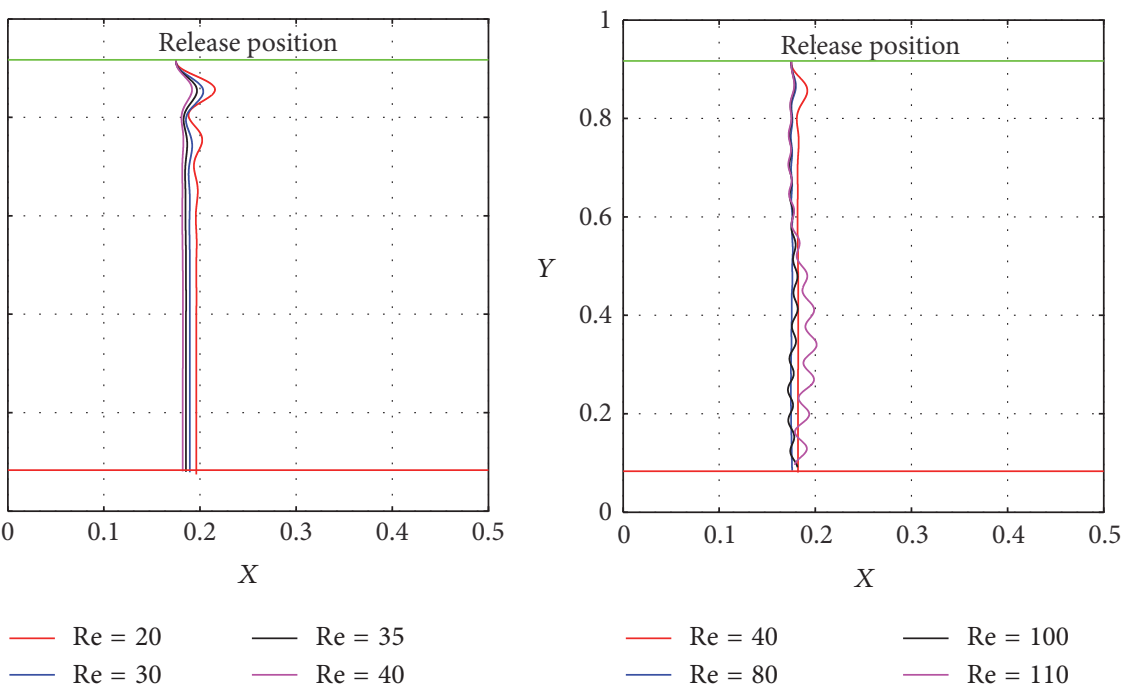

(c)

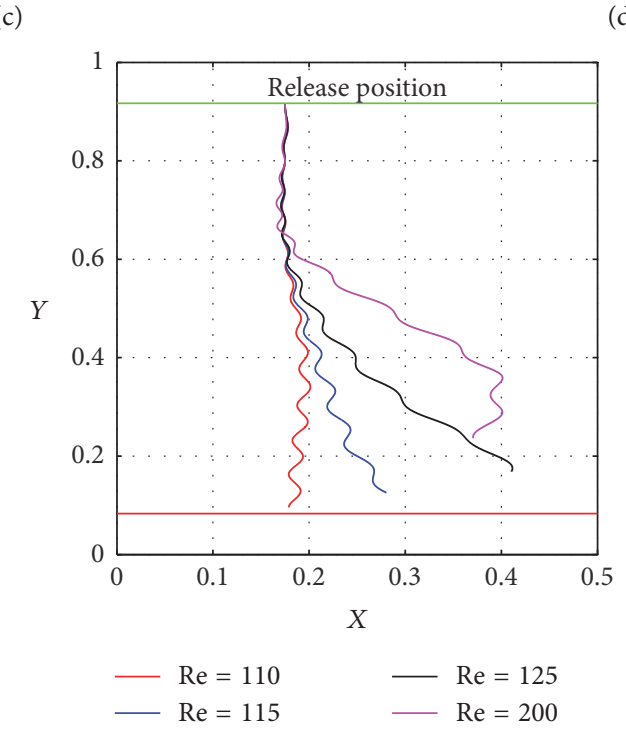

(d)

(e)

FIGURE 11: The trajectory of the left particle at different Reynolds numbers: (a) $\operatorname{Re}=5-10$, (b) $\operatorname{Re}=10-20,(\mathrm{c}) \mathrm{Re}=20-40,(\mathrm{~d}) \mathrm{Re}=40-110$, and (e) $\operatorname{Re}=110-200$. 


\section{Acknowledgments}

This work is supported by the Zhejiang Provincial Natural Science Foundation of China (Grant no. LY15A020004) and the National Natural Science Foundation of China (Grant no. 11272302).

\section{References}

[1] C. S. Peskin, "Numerical analysis of blood flow in the heart," Journal of Computational Physics, vol. 25, no. 3, pp. 220-252, 1977.

[2] R. Mittal and G. Iaccarino, "Immersed boundary methods," Annual Review Fluid Mechanics, vol. 37, pp. 239-261, 2005.

[3] R. Glowinski, T.-W. Pan, T. I. Hesla, and D. D. Joseph, "A distributed Lagrange multiplier/fictitious domain method for particulate flows," International Journal of Multiphase Flow, vol. 25, no. 5, pp. 755-794, 1999.

[4] Z. Yu and X. Shao, "A direct-forcing fictitious domain method for particulate flows," Journal of Computational Physics, vol. 227, no. 1, pp. 292-314, 2007.

[5] D. Nie and J. Lin, "A LB-DF/FD method for particle suspensions," Communications in Computational Physics, vol. 7, no. 3, pp. 544-563, 2010.

[6] D. Nie, Y. Wang, and K. Zhang, "Long-time decay of the translational/rotational velocity autocorrelation function for colloidal particles in two dimensions," Computers and Mathematics with Applications, vol. 61, no. 8, pp. 2152-2157, 2011.

[7] D.-M. Nie, J.-Z. Lin, and K. Zhang, "Flow patterns in the sedimentation of a capsule-shaped particle," Chinese Physics Letters, vol. 29, no. 8, Article ID 084703, 2012.

[8] D. Nie and J. Lin, "A Lattice Boltzmann-direct forcing/fictitious domain model for Brownian particles in fluctuating fluids," Communications in Computational Physics, vol. 9, no. 4, pp. 959-973, 2011.

[9] J. Feng, H. H. Hu, and D. D. Joseph, "Direct simulation of initial value problems for the motion of solid bodies in a Newtonian fluid Part 1. Sedimentation," Journal of Fluid Mechanics, vol. 261, pp. 95-134, 1994.

[10] Z. Xia, K. W. Connington, S. Rapaka, P. Yue, J. J. Feng, and S. Chen, "Flow patterns in the sedimentation of an elliptical particle," Journal of Fluid Mechanics, vol. 625, pp. 249-272, 2009.

[11] C. K. Aidun and E.-J. Ding, "Dynamics of particle sedimentation in a vertical channel: period-doubling bifurcation and chaotic state," Physics of Fluids, vol. 15, no. 6, p. 1612, 2003.

[12] A. El Yacoubi, S. Xu, and Z. J. Wang, "Computational study of the interaction of freely moving particles at intermediate Reynolds numbers," Journal of Fluid Mechanics, vol. 705, pp. 134-148, 2012.

[13] D. Nie, J. Lin, and M. Zheng, "Direct numerical simulation of multiple particles sedimentation at an intermediate Reynolds number," Communications in Computational Physics, vol. 16, no. 3, pp. 675-698, 2014.

[14] L. Wang, Z. L. Guo, and J. C. Mi, "Drafting, kissing and tumbling process of two particles with different sizes," Computers and Fluids, vol. 96, pp. 20-34, 2014.

[15] D. M. Nie, J. Z. Lin, and R. Q. Chen, "Grouping behavior of coaxial settling particles in a narrow channel," Physical Review E, vol. 93, no. 1, Article ID 013114, 2016.

[16] R. Verjus, S. Guillou, A. Ezersky, and J. Angilella, "Chaotic sedimentation of particle pairs in a vertical channel at low
Reynolds number: multiple states and routes to chaos," Physics of Fluids, vol. 28, no. 12, Article ID 123303, 2016.

[17] A. Amiri Delouei, M. Nazari, M. H. Kayhani, S. K. Kang, and S. Succi, "Non-Newtonian particulate flow simulation: a directforcing immersed boundary-lattice Boltzmann approach," Physica A. Statistical Mechanics and Its Applications, vol. 447, pp. 1-20, 2016.

[18] C. K. Aidun, Y. Lu, and E.-J. Ding, "Direct analysis of particulate suspensions with inertia using the discrete Boltzmann equation," Journal of Fluid Mechanics, vol. 373, pp. 287-311, 1998. 


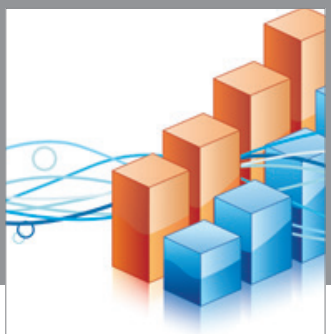

Advances in

Operations Research

vatem alat4

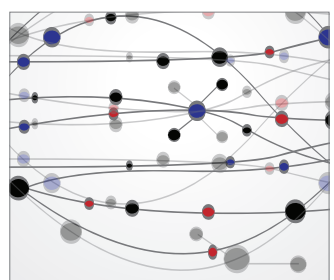

\section{The Scientific} World Journal
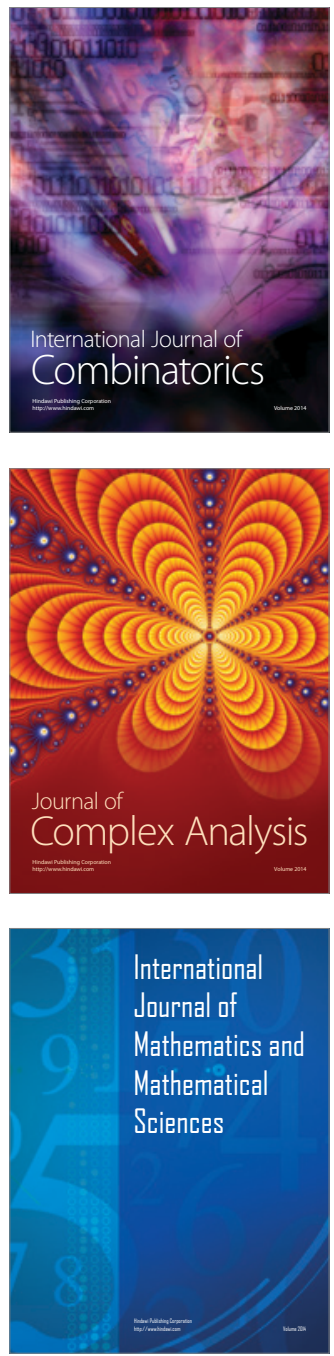
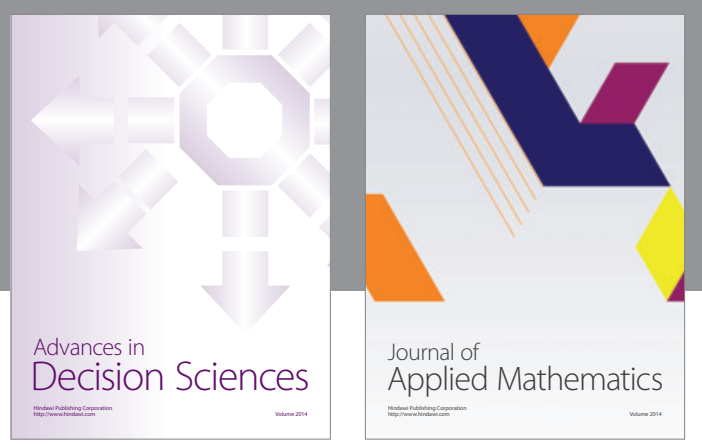

Algebra

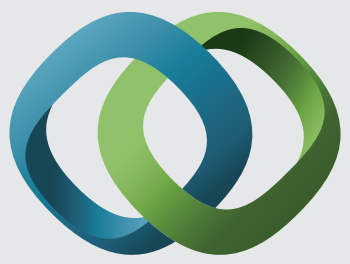

\section{Hindawi}

Submit your manuscripts at

https://www.hindawi.com
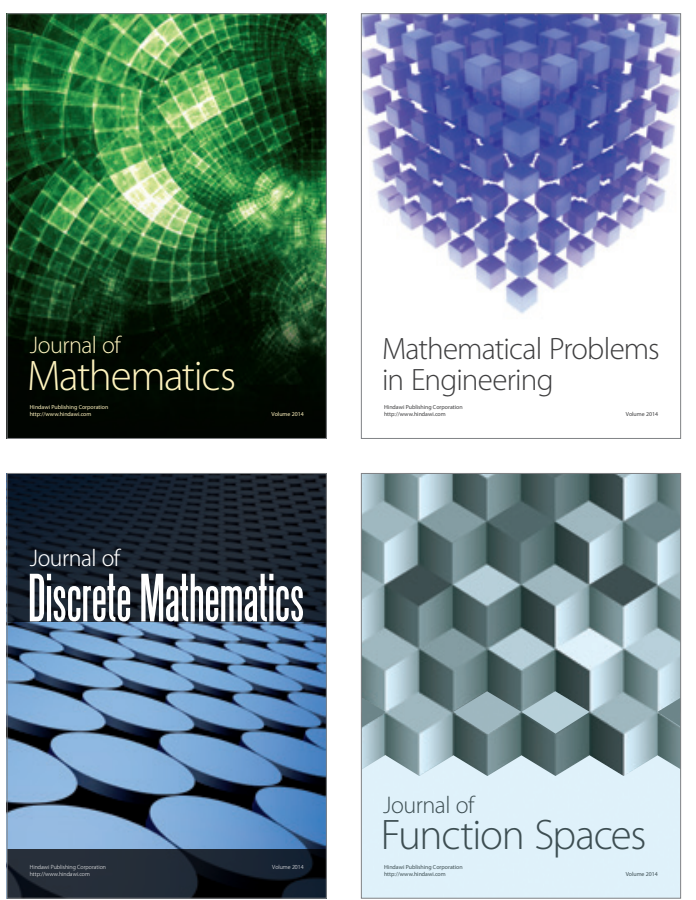

Mathematical Problems in Engineering
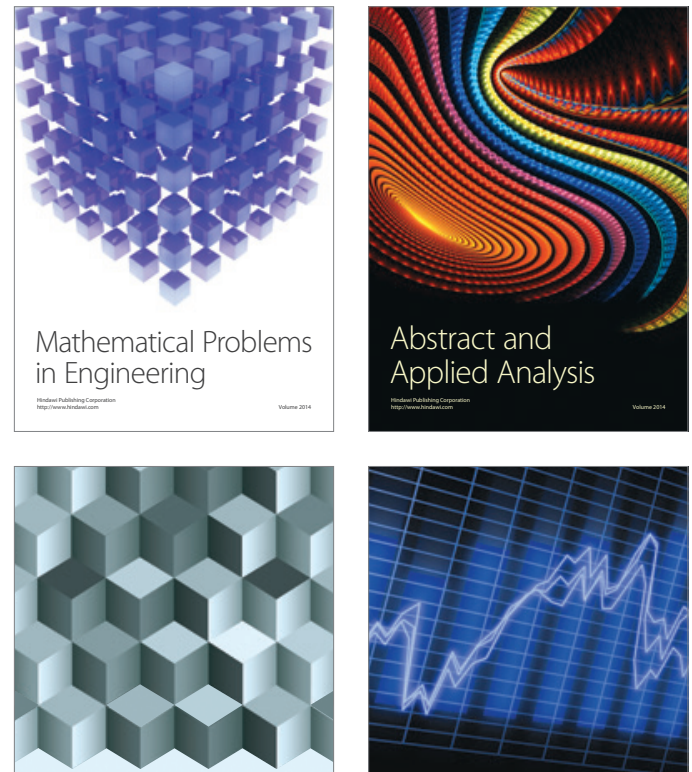

Journal of

Function Spaces

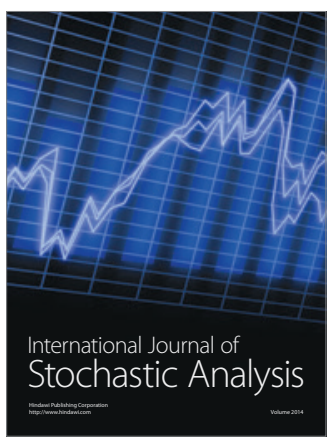

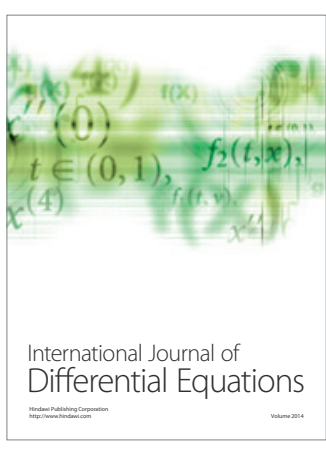
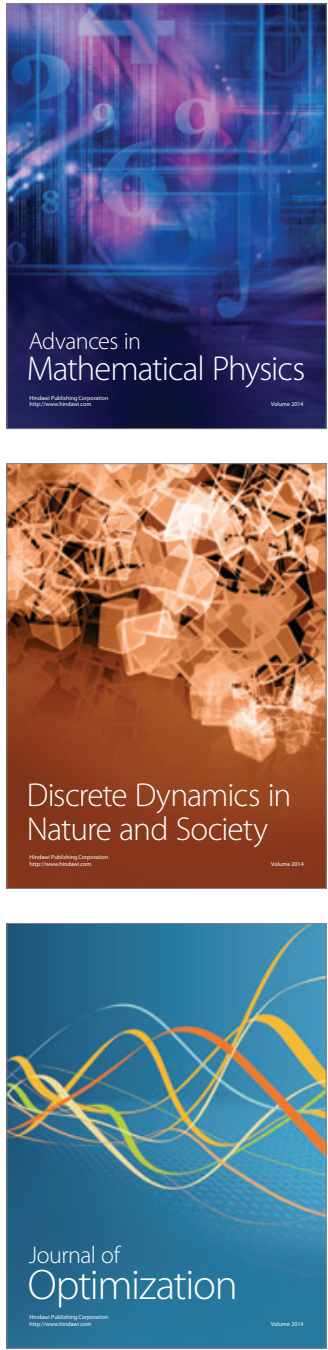